\title{
Mobility enhancement and temperature dependence in top-gated single-layer $\mathrm{MoS}_{2}$
}

\author{
Zhun-Yong Ong and Massimo V. Fischett:* \\ Department of Materials Science and Engineering, University of Texas at Dallas, \\ $800 W$ Campbell Rd RL10, Richardson, TX 75080
}

\begin{abstract}
The deposition of a high- $\kappa$ oxide overlayer is known to significantly enhance the room-temperature electron mobility in single-layer $\mathrm{MoS}_{2}$ (SLM) but not in single-layer graphene (SLG). We give a quantitative account of how this mobility enhancement is due to the non-degeneracy of the twodimensional electron gas system in SLM at accessible temperatures. Using our charged impurity scattering model [Ong and Fischetti, Phys. Rev. B 86, 121409 (2012)] and temperature-dependent polarizability, we calculate the charged impurity-limited mobility $\left(\mu_{\mathrm{imp}}\right)$ in SLM with and without a high- $\kappa\left(\mathrm{HfO}_{2}\right)$ top gate oxide at different electron densities and temperatures. We find that the mobility enhancement is larger at low electron densities and high temperatures because of finite-temperature screening, thus explaining the enhancement of the mobility observed at room temperature. $\mu_{\mathrm{imp}}$ is shown to decrease significantly with increasing temperature, suggesting that the strong temperature dependence of measured mobilities should not be interpreted as being solely due to inelastic scattering with phonons. We also reproduce the recently seen experimental trend in which the temperature scaling exponent $(\gamma)$ of $\mu_{\mathrm{imp}} \propto T^{-\gamma}$ is smaller in top-gated SLM than in bare SLM. Finally, we show that a $\sim 37$ percent mobility enhancement can be achieved by reducing the $\mathrm{HfO}_{2}$ thickness from 20 to $2 \mathrm{~nm}$.
\end{abstract}

\section{INTRODUCTION}

In recent years, two-dimensional metal dichalcogenides have attracted much attention as viable alternatives to graphene [1] for post-CMOS nanoelectronic applications [2]. In particular, single-layer $\mathrm{MoS}_{2}$ (SLM) has been the focus of much research [3] $[3]$. Like single-layer graphene (SLG), SLM is an atomically thin two-dimensional crystal. Given its atomic thickness and close proximity to the substrate, the electron density in SLM can be tuned via a vertical electric field. However, this means SLM is highly susceptible to the local electrical field generated by charged impurities near or at the substrate surface. Therefore, the electron mobility is expected to be strongly affected by charged impurity (CI) scattering 9] and/or remote phonon scattering [10 14].

Radisavljevic and co-workers [3] recently measured the electron mobility $\left(\mu_{e}\right)$ in $\mathrm{SiO}_{2}$-supported SLM to be between 0.1 and $10 \mathrm{~cm}^{2} \mathrm{~V}^{-1} \mathrm{~s}^{-1}$. However, when a thin layer of $\mathrm{HfO}_{2}(\kappa=22)$ was deposited on the SLM to form a top gate, they reported a 20 -fold mobility increase of $\sim 200$ $\mathrm{cm}^{2} \mathrm{~V}^{-1} \mathrm{~s}^{-1}$ at room temperature. More recent and accurate mobility measurements [8] based on the Hall effect yield a maximum mobility of $\sim 63 \mathrm{~cm}^{2} \mathrm{~V}^{-1} \mathrm{~s}^{-1}$ in topgated SLM and $\sim 17 \mathrm{~cm}^{2} \mathrm{~V}^{-1} \mathrm{~s}^{-1}$ in bare uncovered SLM at $260 \mathrm{~K}$, an almost 4-fold improvement. This mobility enhancement was attributed to screening from the $\mathrm{HfO}_{2}$ which reduces CI scattering, believed to be the dominant scattering process. Amani and co-workers also found a similar 3-fold enhancement in $\mathrm{Al}_{2} \mathrm{O}_{3}$-covered SLM grown with chemical vapor deposition [15].

This mobility enhancement from dielectric screening is puzzling given that the same effect has not been seen in top-gated SLG. When Fallahazad and co-workers deposited $\mathrm{HfO}_{2}$ on $\mathrm{SiO}_{2}$-supported SLG, they did not ob- serve any mobility enhancement although they did find that a thinner gate oxide increases the mobility in SLG [16]. This has been explained as consequence of greater screening of the charged impurities by the metal gate [17. In every instance that we know of [16, 18 21], the deposition of an oxide layer on high-mobility, non-epitaxial SLG has lead to a mobility decrease, probably as a result of more CI and defect scattering. Thus, it is surprising to observe a several-fold improvement for SLM. This suggests that CI scattering is qualitatively different in topgated SLM [3]. The variance between the data from Refs. [3] and [16] is striking, and may be due to the different electronic band structures, the nature of the interaction between the substrate and the SLG/SLM, or the type of charge screening. In both cases, the substrate material is $\mathrm{SiO}_{2}$ while the gate oxide is $\mathrm{HfO}_{2}$ (30 nm thick in Ref. [3] and $11 \mathrm{~nm}$ in Ref. [16]), and the mobility measurement methods (two-probe) are similar. This and the similar stack structure rule out the possibility of the difference being due to the top gate capacitance [22, 23].

Another salient feature of electron transport in SLM is that the deposition of the top gate oxide alters the temperature dependence of the electron mobility. At room temperature $(300 \mathrm{~K})$, the phonon-limited electron mobility is predicted to scale as $\mu_{e} \propto T^{-\gamma}$ with $\gamma=1.69$ and $\mu_{e} \approx 410 \mathrm{~cm}^{2} \mathrm{~V}^{-1} \mathrm{~s}^{-1}$ in bare SLM and $\gamma=1.52$ and $\mu_{e} \approx 480 \mathrm{~cm}^{2} \mathrm{~V}^{-1} \mathrm{~s}^{-1}$ in top-gated SLM where the homopolar optical phonon mode is assumed to be quenched 5]. Measurements by Radisavljevic and Kis of the hightemperature $(T=80$ to $280 \mathrm{~K})$ Hall mobility in bare SLM yield $\gamma \approx 1.4$, in good agreement with Ref. [5], although the absolute value of the mobility is about one order of magnitude smaller with $\mu_{e}<20 \mathrm{~cm}^{2} \mathrm{~V}^{-1} \mathrm{~s}^{-1}$ at 260 $\mathrm{K}$ [8]. Their measurements on top-gated SLM also yield $\gamma=0.3$ to 0.73 with $\mu_{e}=57$ to $63.7 \mathrm{~cm}^{2} \mathrm{~V}^{-1} \mathrm{~s}^{-1}$ at 260 $\mathrm{K}$ in samples exhibiting the metal-insulator transition. 
Their bare SLM results are also in good agreement with the more recent data from Baugher and co-workers whose measurements on bare SLM give $\mu_{e}<20 \mathrm{~cm}^{2} \mathrm{~V}^{-1} \mathrm{~s}^{-1}$ and $\gamma=1.7$ at $300 \mathrm{~K}$ [24]. Although experimentally determined values of $\gamma$ from Refs. [8, 24] $(\gamma=1.4$ and 1.7 respectively) agree with the theoretically predicted value of $\gamma=1.69$ in bare SLM, the experimental values $\left(\mu_{e}<20\right.$ $\mathrm{cm}^{2} \mathrm{~V}^{-1} \mathrm{~s}^{-1}$ ) are one order of magnitude smaller than the theoretical value $\left(\mu_{e} \approx 410 \mathrm{~cm}^{2} \mathrm{~V}^{-1} \mathrm{~s}^{-1}\right)$ and suggest that intrinsic phonon scattering is not the dominant factor in the temperature dependence of $\mu_{e}$.

In this article, we study temperature-dependent, charged impurity-limited electron transport in bare and top-gated single-layer $\mathrm{MoS}_{2}$ by adapting the model developed in Ref. [17] and including not only the effect of the dielectric environment but also the temperature dependence of the charge polarizability. $\mathrm{HfO}_{2}$-covered SLM on a $\mathrm{SiO}_{2}$ substrate is used as a model system here although the theory can be easily generalized to other gate dielectrics and single-layer transition metal dichalcogenides (TMDs). Our use of the temperature-dependent charge polarizability is motivated by the electron transport data from Ghatak and co-workers [25], which have been interpreted to imply that charged impurities are weakly screened at room temperature. For simplicity, electron-phonon interaction is mostly ignored here to isolate the effects of screening by the charge polarizability as well as the dielectric environment although scattering with the intrinsic phonons is included when it comes to the mobility scaling with temperature. The difference between the charge impurity-limited electron mobility $\left(\mu_{\mathrm{imp}}\right)$ in bare and top-gated SLM at different temperatures $(T)$ and electron densities $(n)$ is used to explain the screening effect of the gate oxide on room-temperature electron transport. We also show that the lower mobility at higher temperatures can be due to temperaturedependent screening. Lastly, we predict the scaling of $\mu_{\text {imp }}$ with the gate oxide thickness $\left(t_{\mathrm{ox}}\right)$ at room temperature.

\section{METHODOLOGY}

\section{A. Charged impurity scattering potential}

A schematic of the setup is shown in Fig. 11 The model consists of a SLM sheet sandwiched between two oxide layers with the interface at $z=0$ on the $x-y$ plane. The substrate oxide $\left(\mathrm{SiO}_{2}\right)$ is semi-infinite $(z<0)$ while the gate oxide has a thickness of $t_{\mathrm{ox}}$ (i.e. $0 \leq z<t_{\mathrm{ox}}$ ). We approximate SLM as an ideal zero-thickness twodimensional electron gas (2DEG). To determine $\mu_{\mathrm{imp}}$, we compute the scattering rate $\Gamma_{\text {imp }}$ for the single CI scattering potential $\phi_{q}^{\text {scr }}(0)$. The expression for the $\phi_{q}^{\text {scr }}(0)$ is

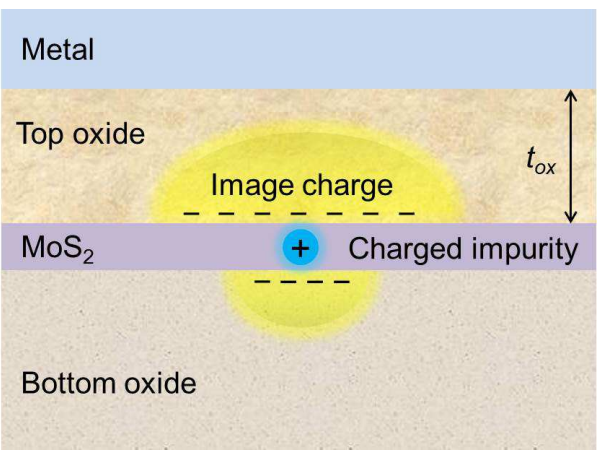

Figure 1: (Color online) Basic model used in our calculation. The SLM is an infinitely thin layer at the interface $(z=0)$ between a semi-infinite substrate and a top oxide layer of thickness $t_{\mathrm{ox}}$. The dielectric is capped with metal, which we assume to be a perfect conductor. The charged impurity at the interface has image charges under and above it in the substrate and top gate, respectively.

17]:

$$
\phi_{q}^{\mathrm{scr}}(z=0)=\frac{e^{2} G_{q}(0,0)}{\epsilon_{2 \mathrm{D}}(q, T)}
$$

where $\mathbf{q}, e$ and $G_{q}(0,0)$ are the wave vector, the absolute electron charge quantum, and the Fourier transform (with respect to $x$ and $y$ ) of the Green's function solution of the Poisson equation, respectively; $\epsilon_{2 \mathrm{D}}(q, T)$ is the generalized static dielectric function, given by $\epsilon_{2 \mathrm{D}}(q, T)=1-e^{2} G_{q}(0,0) \Pi\left(q, T, E_{F}\right)$ where $\Pi\left(q, T, E_{F}\right)$ is the temperature-dependent static charge polarizability. The expression for $G_{q}(0,0)$ is $G_{q}(0,0)=$ $\left\{\left[\epsilon_{\mathrm{tox}}^{0} \operatorname{coth}\left(q t_{\mathrm{ox}}\right)+\epsilon_{\mathrm{box}}^{0}\right] q\right\}^{-1}$ where $\epsilon_{\mathrm{tox}}^{0}$ and $\epsilon_{\mathrm{box}}^{0}$ are the static permittivity of the top and bottom oxides, respectively. The electrostatic boundary conditions are included in $G_{q}(0,0)$.

\section{B. Fermi temperature and temperature-dependent screening}

While graphene remains degenerate even at low density around room temperature, in TMDs the temperature dependence of the dielectric response can play a significant role. We take it into account by first examining the long-wavelength, finite-temperature approximation for $\Pi\left(q, T, E_{F}\right)[26]$, i.e.

$$
\lim _{q \rightarrow 0} \Pi\left(q, T, E_{F}\right)=-\frac{g m_{\mathrm{eff}}}{2 \pi \hbar^{2}}\left[1-\exp \left(\frac{-\pi \hbar^{2} n}{2 m_{\mathrm{eff}} k_{B} T}\right)\right],
$$

where $g$ and $m_{\text {eff }}$ are the valley-spin degeneracy $(g=4)$ and the effective electron mass, respectively; $E_{F}$ is the chemical potential and is related to $n$ via the equation $E_{F}=k_{B} T \ln \left\{\exp \left[\pi \hbar^{2} n /\left(2 m_{\mathrm{eff}} k_{B} T\right)\right]-1\right\} ; k_{B}$ and $\hbar$ are the Boltzmann and Planck constant, respectively. For 
a given electron density $n$, the 2DEG can be considered degenerate when $T \ll T_{F}$ where $T_{F}=\pi \hbar^{2} n /\left(2 m_{\mathrm{eff}} k_{B}\right)$ is the characteristic Fermi temperature. At $n=10^{12} \mathrm{~cm}^{-2}$, $T_{F}=29 \mathrm{~K}$. Therefore, we need to use finite-temperature screening for the range of electron densities and temperatures in our calculations later. At finite $q$, we can use the more general expression [27 29]:

$$
\Pi\left(q, T, E_{F}\right)=\int_{0}^{\infty} d \mu \frac{\Pi(q, 0, \mu)}{4 k_{B} T \cosh ^{2}\left(\frac{E_{F}-\mu}{2 k_{B} T}\right)},
$$

where $\Pi(q, 0, \mu)=\Pi(0,0, \mu)\left\{1-\Theta\left(q-2 k_{F}\right)[1-\right.$ $\left.\left.\left(2 k_{F} / q\right)^{2}\right]^{1 / 2}\right\}$ with $k_{F}=\sqrt{2 m_{\mathrm{eff}} \mu} / \hbar$ and $\Pi(0,0, \mu)=$ $-g m_{\mathrm{eff}} /\left(2 \pi \hbar^{2}\right)$. Figure 2 shows the $q$-dependence of $\Pi\left(q, T, E_{F}\right)$ at $T=0,50,100$ and $300 \mathrm{~K}$ for (a) $n=10^{12}$ $\mathrm{cm}^{-2}$ and (b) $n=10^{13} \mathrm{~cm}^{-2}$. For the same given $T$, the change in the polarizability relative to the $0 \mathrm{~K}$ case is greater at $n=10^{12} \mathrm{~cm}^{-2}\left(T_{F}=29 \mathrm{~K}\right)$ than at $n=10^{13}$ $\mathrm{cm}^{-2}\left(T_{F}=290 \mathrm{~K}\right)$. We also observe that $\Pi\left(q, T, E_{F}\right)$ is significantly smaller at $300 \mathrm{~K}$ than at $0 \mathrm{~K}$. In general, $\Pi\left(q, T, E_{F}\right)$ in Eq. (2), which appears in the denominator in Eq. (11) and corresponds to charge screening, vanishes as $n \rightarrow 0$ or $T \rightarrow \infty$, i.e. charge screening weakens with decreasing electron density or increasing temperature. Hence, the CI scattering strength increases as $n \rightarrow 0$ or $T \rightarrow \infty$. To illustrate this, we plot the corresponding scattering potential $\phi_{q}^{\text {scr }}$ in top-gated SLM at $T=0,50,100$ and $300 \mathrm{~K}$, normalized to $\phi_{q=0}^{\text {scr }}$ at $T=0$ K, in Fig. 2 for (c) $n=10^{12} \mathrm{~cm}^{-2}$ and (d) $n=10^{13} \mathrm{~cm}^{-2}$. For $n=10^{13} \mathrm{~cm}^{-2}$, the scattering potential remains relatively unchanged as $T$ increases, unlike the scattering potential for $n=10^{12} \mathrm{~cm}^{-2}$ which increases by up to an order of magnitude as $T$ increases from $0 \mathrm{~K}$ to 300 $\mathrm{K}$, because the Fermi temperature at $n=10^{13} \mathrm{~cm}^{-2}$ is $T_{F}=290 \mathrm{~K}$.

Following Ref. [5] we approximate the electron dispersion in SLM with a parabolic expression $E(\mathbf{k})=$ $\hbar^{2} k^{2} /\left(2 m_{\text {eff }}\right)$ with effective mass $m_{\text {eff }}=0.48 m_{0}$ (where $m_{0}$ is the free electron mass) and minimum at the symmetry point $K$. The use of a single valley should not constitute a big error since at low fields no interband transitions are expected to take place [5]. The expression for the CI scattering rate is [17]:

$$
\begin{aligned}
\Gamma_{\mathrm{imp}}\left(E_{\mathbf{k}}\right)= & \frac{n_{\mathrm{imp}}}{2 \pi \hbar} \int d \mathbf{k}^{\prime}\left|\phi_{\left|\mathbf{k}-\mathbf{k}^{\prime}\right|}^{\mathrm{scr}}(d)\right|^{2} \\
& \times\left(1-\cos \theta_{\mathbf{k} \mathbf{k}^{\prime}}\right) \delta\left(E_{\mathbf{k}}-E_{\mathbf{k}^{\prime}}\right),
\end{aligned}
$$

where $\theta_{\mathbf{k k}^{\prime}}$ is the scattering angle between the $\mathbf{k}$ and $\mathbf{k}^{\prime}$ states, and $n_{\mathrm{imp}}$ is the CI concentration which is a fitting parameter. The expression for the CI-limited electron mobility is:

$$
\mu_{\mathrm{imp}}=\frac{e}{\pi \hbar^{2} k_{B} T} \int_{0}^{\infty} f(E)[1-f(E)] \Gamma_{\mathrm{imp}}(E)^{-1} E d E,
$$

where $f(E)$ is the equilibrium Fermi-Dirac distribution function. By using Eq. (5), we assume that electron
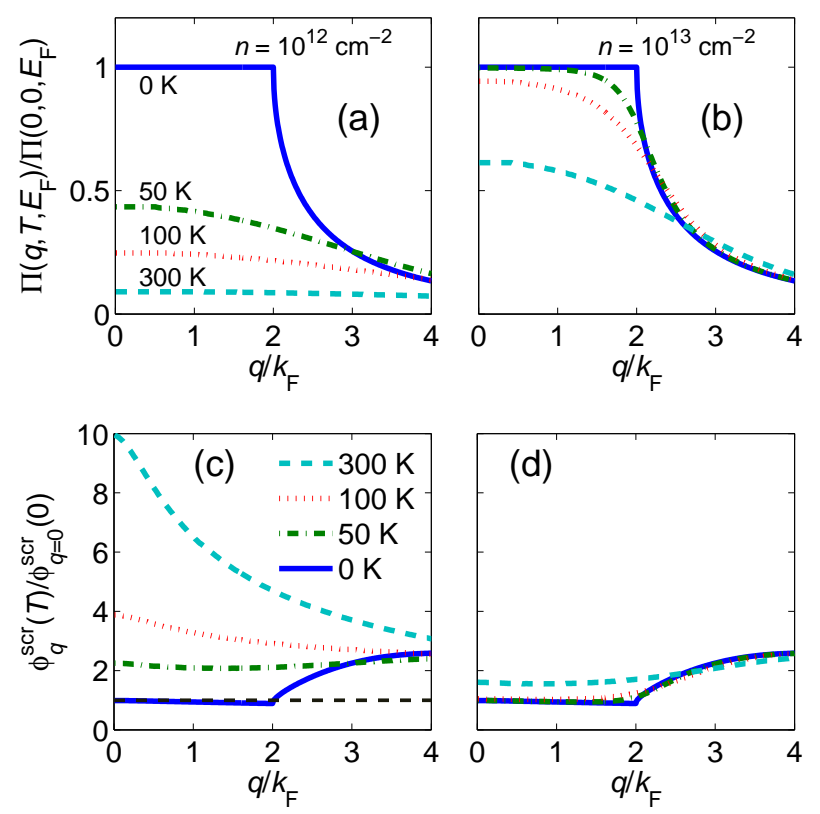

Figure 2: (Color online) Plot of the normalized polarizability $\Pi\left(q, T, E_{F}\right) / \Pi\left(0,0, E_{F}\right)$ for (a) $n=10^{12} \mathrm{~cm}^{-2}$ and (b) $n=10^{13} \mathrm{~cm}^{-2}$ at $T=0 \mathrm{~K}$ (solid), $50 \mathrm{~K}$ (dash-dot), $100 \mathrm{~K}$ (dotted) and $300 \mathrm{~K}$ (dashed). We also plot the corresponding normalized scattering potential $\phi_{q}^{\mathrm{scr}}(T) / \phi_{q=0}^{\mathrm{scr}}(T=0)$ for $(\mathrm{c})$ $n=10^{12} \mathrm{~cm}^{-2}$ and (d) $n=10^{13} \mathrm{~cm}^{-2}$ in top-gated SLM.

transport is described by semiclassical band transport, as in Refs. [5, 30] and opposed to hopping transport as suggested in Ref. [25], and that the dominant scattering mechanism is CI scattering, which is mostly at the Fermi surface. The main momentum relaxation process corresponds to the momentum change of $q \sim 2 k_{F}$ and the related Fourier component of the scattering potential $\phi_{2 k_{F}}^{\mathrm{scr}}$, which is inversely proportional to the dielectric function $\epsilon_{2 \mathrm{D}}\left(2 k_{F}, T\right)$ and strongly affected by the temperature broadening of $\Pi\left(2 k_{F}, T, E_{F}\right)$ [28, 31].

\section{RESULTS AND DISCUSSION}

\section{A. Electron density dependence of mobility at low and high temperature}

The variables $\mu_{\mathrm{imp}}^{0}$ and $\mu_{\mathrm{imp}}^{\mathrm{TG}}$ denote the CI-limited mobility in bare $\mathrm{SiO}_{2}$-supported $\left(\epsilon_{\text {tox }}^{0}=\epsilon_{0}\right.$ and $\left.t_{\text {ox }}=\infty\right)$ and 30-nm- $\mathrm{HfO}_{2}$-top-gated, $\mathrm{SiO}_{2}$-supported $\left(\epsilon_{\text {tox }}^{0}=22 \epsilon_{0}\right.$ and $\left.t_{\mathrm{ox}}=30 \mathrm{~nm}\right)$ SLM. We assume $n_{\mathrm{imp}}=4 \times 10^{12} \mathrm{~cm}^{-2}$ for ease of comparison with the results in Ref. [3]. At $n=2 \times 10^{13} \mathrm{~cm}^{-2}$ and $T=10 \mathrm{~K}$, this yields $\mu_{\mathrm{imp}}^{\mathrm{TG}} \sim 150$ $\mathrm{cm}^{2} \mathrm{~V}^{-1} \mathrm{~s}^{-1}$, comparable to that measured by Radisavljevic and Kis [8] at low temperatures. We first calculate and plot in Fig. $3 \mu_{\mathrm{imp}}^{0}$ and $\mu_{\mathrm{imp}}^{\mathrm{TG}}$ at $T=10 \mathrm{~K}$, from $n=10^{12}$ to $2 \times 10^{13} \mathrm{~cm}^{-2}$ in steps of $\Delta n=10^{12} \mathrm{~cm}^{-2}$. 


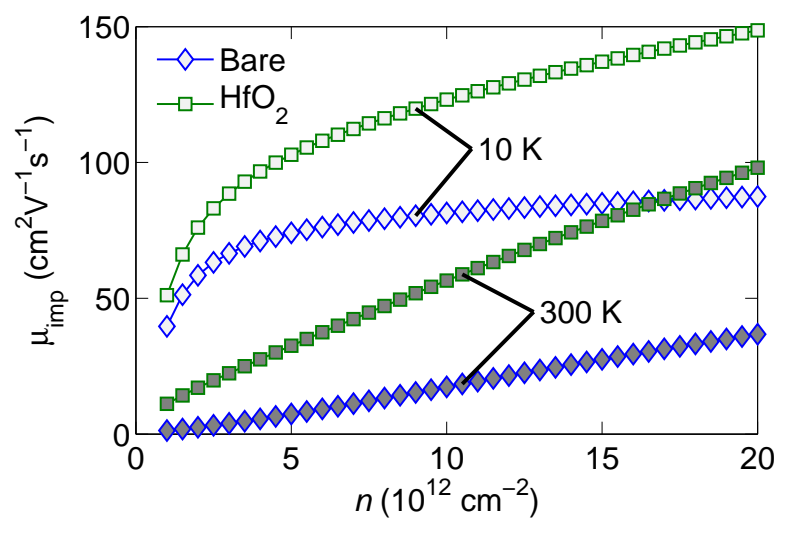

Figure 3: (Color online) Plot of $\mu_{\mathrm{imp}}^{0}$ ('Bare') and $\mu_{\mathrm{imp}}^{\mathrm{TG}}$ (' $\mathrm{HfO}_{2}$ ') at $T=10 \mathrm{~K}$ (hollow symbols) and $T=300 \mathrm{~K}$ (solid symbols) for $n=10^{12}$ to $2 \times 10^{13} \mathrm{~cm}^{-2}$ for $n_{\text {imp }}=4.0 \times 10^{12}$ $\mathrm{cm}^{-2}$. At $300 \mathrm{~K}$, the mobility scales almost linearly with the electron density.

The corresponding Fermi temperature range is $T_{F}=29$ to $580 \mathrm{~K}$. We find that both $\mu_{\mathrm{imp}}^{0}$ and $\mu_{\mathrm{imp}}^{\mathrm{TG}}$ increase monotonically with $n$, in good agreement with the Hall mobility data given in Ref. 24], with the density dependence stronger for $\mu_{\mathrm{imp}}^{\mathrm{TG}}$. At low densities $\left(n<4 \times 10^{12} \mathrm{~cm}^{-2}\right)$, the density dependence is markedly greater.

Our results indicate that $\mu_{\mathrm{imp}}^{\mathrm{TG}}$ is higher than $\mu_{\mathrm{imp}}^{0}$, with the relative difference increasing with $n$; at $n=10^{12}$ $\mathrm{cm}^{-2}$, we have $\mu_{\mathrm{imp}}^{\mathrm{TG}} / \mu_{\mathrm{imp}}^{0}=1.29$ while at $n=2 \times 10^{13}$ $\mathrm{cm}^{-2}$, we have $\mu_{\mathrm{imp}}^{\mathrm{TG}} / \mu_{\mathrm{imp}}^{0}=1.70$. This suggests that the mobility enhancement from overlaying SLM with a high$\kappa$ material is modest at low temperatures. This is because at low temperatures $\left(T \ll T_{F}\right)$, screening is dominated by the charge polarizability. To see how, we rewrite the scattering potential of a single CI in Eq. (11) as:

$$
\phi_{q}^{\mathrm{scr}}(0)=\frac{e^{2} G_{q}(0,0)}{1-e^{2} G_{q}(0,0) \Pi\left(q, T, E_{F}\right)} .
$$

In the long-wavelength limit, the second term in the denominator, which corresponds to the screening charge, dominates, giving us $\lim _{q \rightarrow 0} \phi_{q}^{\text {scr }}(0)=-\Pi(q=$ $\left.0, T, E_{F}\right)^{-1}$. Thus, the scattering potential is independent of the dielectric environment in the long-wavelength limit and depends only on the polarizability. At low $T$ and $q<2 k_{F}$, the polarizability is nearly independent of q, i.e. $\lim _{T \rightarrow 0} \Pi\left(q<2 k_{F}, T, E_{F}\right)=-2 m_{\mathrm{eff}} /\left(\pi \hbar^{2}\right)$. This explains why $\mu_{\mathrm{imp}}^{\mathrm{TG}} / \mu_{\mathrm{imp}}^{0}$ is close to unity. The decrease of $\mu_{\mathrm{imp}}^{0}$ and $\mu_{\mathrm{imp}}^{\mathrm{TG}}$ with smaller $n$ is due to the fact that at small $n$, we have $\lim _{n \rightarrow 0} \Pi\left(q, T, E_{F}\right) \propto n$ which implies that the scattering potential strength scales as $\sim n^{-1}$.

On the other hand, experimental measurements reveal that covering SLM with a high- $\kappa$ dielectric leads to significant room-temperature mobility enhancement [8]. This suggests that screening by the dielectric plays a greater role in the temperature regime $T \gtrsim T_{F}$. Hence, the

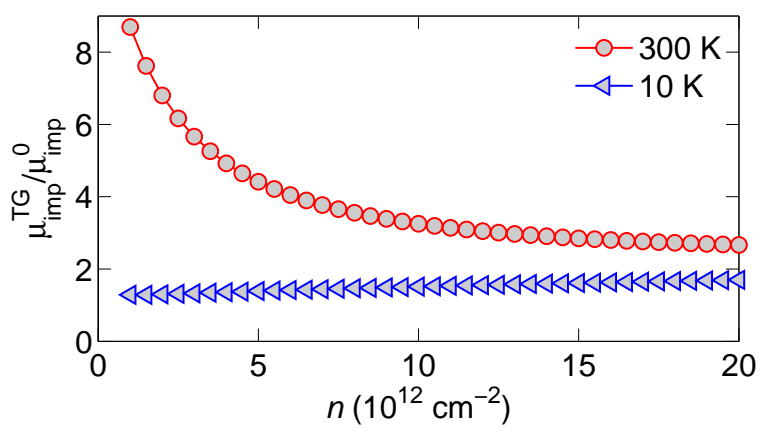

Figure 4: (Color online) Plot of the mobility enhancement $\mu_{\mathrm{imp}}^{\mathrm{TG}} / \mu_{\mathrm{imp}}^{0}$ at $10 \mathrm{~K}$ (circle) and $300 \mathrm{~K}$ (triangle). The mobility enhancement increases at higher temperatures or lower electron densities.

screening effect of the charge polarizability in SLM is less significant. To show this, we repeat our calculation of $\mu_{\mathrm{imp}}^{0}$ and $\mu_{\mathrm{imp}}^{\mathrm{TG}}$ but now at room temperature $(300 \mathrm{~K})$. The room-temperature results are also shown in Fig. 3. In contrast to the low-temperature results in Fig. 3, $\mu_{\mathrm{imp}}^{0}$ and $\mu_{\mathrm{imp}}^{\mathrm{TG}}$ are strongly density-dependent and scale almost linearly with $n$, in good agreement with the room-temperature data for bare SLM by Ghatak and co-workers [25]. The linear density-dependence is a signature of weak or absent screening by the polarization charge in SLM. Thus, the role of screening by the surrounding dielectric media becomes more important. At low $n, \mu_{\mathrm{imp}}^{\mathrm{TG}}$ is significantly larger than $\mu_{\mathrm{imp}}^{0}$. At $n=10^{12} \mathrm{~cm}^{-2}, \mu_{\mathrm{imp}}^{0} \approx 1.3 \mathrm{~cm}^{2} \mathrm{~V}^{-1} \mathrm{~s}^{-1}$ while $\mu_{\mathrm{imp}}^{\mathrm{TG}} \approx 11.2$ $\mathrm{cm}^{2} \mathrm{~V}^{-1} \mathrm{~s}^{-1}$, nearly an order-of-magnitude increase. This agrees very well with the measured several-fold mobility enhancement reported in Refs. [8, 15]. We plot the mobility enhancement $\mu_{\mathrm{imp}}^{\mathrm{TG}} / \mu_{\mathrm{imp}}^{0}$ in Fig. 团 at 10 and $300 \mathrm{~K}$. The mobility enhancement is much greater at $300 \mathrm{~K}$ than at $10 \mathrm{~K}$ because of the temperature-induced weakening of the charge polarizability. At $300 \mathrm{~K}$, the mobility enhancement decreases and converges to that at $10 \mathrm{~K}$ as $n$ increases because charge screening becomes stronger at higher densities.

\section{B. Temperature dependence of electron mobility}

The temperature dependence of the electron mobility in experiments is often used to determine the nature of electron transport in semiconductors. When the mobility decreases with increasing $T$, it is commonly interpreted to be a signature of phonon-limited electron transport in the metallic phase [5, 8, 24, 32]; in the insulating phase, the rise in mobility with increasing $T$ is usually characterized as originating from hopping transport [25]. Kaasbjerg and co-workers predict the intrinsic phonon-limited mobility to vary as $\mu_{e} \propto T^{-\gamma}(\gamma=1.52)$ in top-gated SLM. Measurements of $\gamma$ by Radisavljevic and Kis have 
it varying between 0.3 and 0.73 [8], which is suggestive of phonon-limited transport. For ease of comparison, we summarize the representative theoretical and experimental mobility results from Refs. [5, 8, 24, 30] in Table!, together with our results. However, Li and co-workers [30] and Kaasbjerg and co-workers [5] predict the $K$ valleydominated, intrinsic phonon-limited mobility values to be around several hundred $\mathrm{cm}^{2} \mathrm{~V}^{-1} \mathrm{~s}^{-1}$ at room temperature, which are at least an order-of-magnitude larger than measurements [8, 24]. Thus, the temperature dependence of the measured mobility is probably due to extrinsic factors such as charged impurities and remote phonons.

The disparity between our calculated low- and roomtemperature $\mu_{\mathrm{imp}}$ implies that CI scattering is strongly temperature-dependent and plays an important role in the overall mobility temperature dependence. Hence, it is important to quantify the temperature dependence in our model for direct comparison with experiments, in order to understand the causes of this temperature dependence. In particular, we are interested in the temperature scaling of the high-temperature electron mobility $\left(\mu_{e} \propto T^{-\gamma}\right)$, which has been investigated theoretically and experimentally in Refs. [5, 8, 24, 30], and the difference in this temperature scaling between bare and topgated SLM. Radisavljevic and Kis recently reported a substantial decrease in $\gamma$, from $\gamma=1.47$ in bare SLM to $\gamma=0.3-0.73$ in top-gated SLM [8], much greater than that expected from the quenching of homopolar optical phonons [5]. By studying the difference in the temperature dependence of the mobility in bare and top-gated SLM with our model, we hope to shed light on this phenomenon.

Since the temperature variation of the electron mobility may depend on scattering with phonons, we compute the CI/phonon-limited electron mobility $\mu_{e}=\mu_{\mathrm{imp}+\text { phon }}$, taking into account charged impurity as well as intrinsic phonon scattering, in addition to the computation of the CI-limited mobility $\mu_{\mathrm{imp}}$. The intrinsic electron-phonon interactions include the longitudinal acoustic (LA), the transverse acoustic (TA), the intervalley longitudinal optical (LO) and the intravalley homopolar optical (HP) phonons, with the scattering rate formulas and parameters taken directly from Ref. [5]. In our calculation of the $\mathrm{CI} /$ phonon-limited electron mobility $\left(\mu_{\mathrm{imp}+\mathrm{phon}}^{0}\right)$ in bare SLM, we include electron scattering with the LA, TA, LO and HP phonons while in top-gated SLM, we assume that the HP phonons are quenched (as in Ref. [5]) and we do not include them in our calculation of the mobility $\left(\mu_{\mathrm{imp}+\mathrm{phon}}^{\mathrm{TG}}\right)$.

Figure 5 shows (a) $\mu_{\mathrm{imp}+\text { phon }}^{0}$ and (b) $\mu_{\mathrm{imp}+\mathrm{phon}}^{\mathrm{TG}}$ for $n=10^{12}$ to $5 \times 10^{12} \mathrm{~cm}^{-2}$ in steps of $\Delta n=10^{12}$ $\mathrm{cm}^{-2}$, which we take to be representative of the lowdensity regime, and $n=10^{13}$ to $2 \times 10^{13} \mathrm{~cm}^{-2}$ in steps of $\Delta n=2 \times 10^{12} \mathrm{~cm}^{-2}$, which we take to be representative of the high-density 'metallic' regime, from $T=10$ to 300 K. Figures 5 (a) and (b) show that the relative variation of the mobility with $T$ increases as $n$ becomes smaller. The decrease in $\mu_{\mathrm{imp}+\text { phon }}^{0}$ is very large as we go from 10 to 300 K. For example, at $n=10^{12} \mathrm{~cm}^{-2}, \mu_{\mathrm{imp}+\text { phon }}^{0}$ decreases by $>97$ percent. The sensitivity to changes in temperature is significantly greater for $\mu_{\mathrm{imp}+\mathrm{phon}}^{0}$ than $\mu_{\mathrm{imp}}^{\mathrm{TG}}$ phon . The corresponding results for the CI-limited mobilities $\left(\mu_{\mathrm{imp}}^{0}\right.$ and $\left.\mu_{\mathrm{imp}}^{\mathrm{TG}}\right)$ are not shown here since they exhibit a similar trend with respect to temperature change.

From $T=200$ to $300 \mathrm{~K}$ and $n=10^{13}$ to $2 \times 10^{13}$ $\mathrm{cm}^{-2}, \mu_{\mathrm{imp}+\text { phon }}^{0}$ and $\mu_{\mathrm{imp}+\text { phon }}^{\mathrm{TG}}$ exhibit a power-law dependence on $T$, i.e. $\mu_{\mathrm{imp}+\text { phon }} \propto T^{-\gamma}$, similar to that reported in Refs. [8, 24]. We plot $\gamma$ as a function of $n$ for $\mu_{\mathrm{imp}+\mathrm{phon}}^{0}, \mu_{\mathrm{imp}+\mathrm{phon}}^{\mathrm{TG}}, \mu_{\mathrm{imp}}^{0}$ and $\mu_{\mathrm{imp}}^{\mathrm{TG}}$ in Fig. 5(c). The exponent $\gamma$ decreases with $n$ and is also much larger for $\mu_{\mathrm{imp}+\text { phon }}^{0}(\gamma=0.75$ to 1.0$)$ than for $\mu_{\mathrm{imp}+\text { phon }}^{\mathrm{TG}}(\gamma=0.43$ to 0.47), in excellent agreement with Ref. [8] where a significant decrease in $\gamma$ was found for top-gated SLM. The $\gamma$ values for $\mu_{\mathrm{imp}+\text { phon }}^{0}$ are comparable to the $T^{-1}$ behavior expected for a dilute, high-temperature 2DEG [31] but lower than the $\gamma=1.7$ and 1.4 from Refs. 24] and [8], respectively. The values for $\mu_{\mathrm{imp}+\text { phon }}^{\mathrm{TG}}$ are however within the range measured for top-gated SLM samples $(\gamma=0.3$ to 0.73$)$ [8]. The range of $\gamma$ values for the CI-limited mobilities $\mu_{\mathrm{imp}}^{0}$ and $\mu_{\mathrm{imp}}^{\mathrm{TG}}$ are slightly smaller ( 0.70 to 0.98 and 0.30 to 0.36 respectively in the case of $\mu_{\mathrm{imp}}^{0}$ and $\left.\mu_{\mathrm{imp}}^{\mathrm{TG}}\right)$ since the temperature dependence only comes from the finite-temperature charge polarizability. Nevertheless, we observe a similar decrease in $\gamma$ when comparing $\mu_{\mathrm{imp}}^{\mathrm{TG}}$ to $\mu_{\mathrm{imp}}^{0}$. This implies that the change in $\gamma$ is due to the modification of CI scattering in top-gated SLM.

In Ref. [8], $\gamma$ increases with $n$ (from $\gamma=0.55$ at $n=0.76 \times 10^{13} \mathrm{~cm}^{-2}$ to $\gamma=0.78$ at $n=1.35 \times 10^{13}$ $\mathrm{cm}^{-2}$ ) in contrast to our results for $\mu_{\mathrm{imp}}^{\mathrm{TG}}$ and $\mu_{\mathrm{imp}}^{\mathrm{TG}}+$ phon where $\gamma$ decreases as $n$ increases. This suggests that other more strongly temperature-dependent scattering processes may be involved. In Fig. 3(a) and (b), $\mu_{\mathrm{imp}}^{\mathrm{TG}}$ increases with $n$, i.e. CI scattering becomes less important at higher densities. Hence, the relative contribution of the other scattering processes may become more significant.

\section{Gate Oxide Thickness Dependence}

Having shown that screening by the top gate enhances the mobility at room temperature and low $n$ i.e. when $T \gg T_{F}$, we explore the possibility of using a thinner gate oxide to screen the charged impurities. We compute $\mu_{\mathrm{imp}}^{\mathrm{TG}}$ for $n=10^{12}$ to $5 \times 10^{12} \mathrm{~cm}^{-2}$ and $t_{\mathrm{ox}}=2$ to $20 \mathrm{~nm}$ at $300 \mathrm{~K}$. Figure 6 shows the calculated $\mu_{\mathrm{imp}}^{\mathrm{TG}}$ values normalized to the $\mu_{\mathrm{imp}}^{\mathrm{TG}}$ for a semi-infinite top oxide layer. As expected, $\mu_{\mathrm{imp}}^{\mathrm{TG}}$ increases as $t_{\mathrm{ox}}$ decreases because a thin- 


\begin{tabular}{lccccccc}
\hline & & & \multicolumn{2}{c}{ Bare } & \multicolumn{3}{c}{ Top-gated } \\
\cline { 4 - 7 } Reference & $T(\mathrm{~K})$ & Method & $\mu_{e}$ & $\gamma$ & $\mu_{e}$ & $\gamma$ \\
\hline \hline Kaasbjerg et al. (phonon-limited) [5] & 300 & Theory & 410 & 1.69 & 480 & 1.52 \\
Li et al. (phonon-limited) [30] & 300 & Theory & 320 & - & - & - \\
Baugher et al. [24] & 300 & Expt. & $<20$ & 1.7 & - & - \\
Radisavljevic and Kis [8] & 260 & Expt. & 17.2 & 1.4 & 56.9 to 63 & 0.3 to 0.73 \\
Ong and Fischetti (CI-limited) & \multirow{2}{*}{300} & Theory & 17.4 & 0.98 & 56.5 & 0.36 \\
Ong and Fischetti (CI/phonon-limited) & & & 16.2 & 1.0 & 48.9 & 0.46 \\
\hline
\end{tabular}

Table I: Comparison of representative electron mobility $\mu_{e}$ (in units of $\mathrm{cm}^{2} \mathrm{~V}^{-1} \mathrm{~s}^{-1}$ ) and power-law exponent $\gamma\left(\right.$ where $\mu_{e} \propto T^{-\gamma}$ ) values for bare and $\mathrm{HfO}_{2}$ top-gated SLM from Refs. [5, 8, 24, 30]. The results from Li et al. [30] and Kaasbjerg et al. [5] assume $K$ valley-dominated, intrinsic phonon-limited electron transport. The CI-limited results by Ong and Fischetti are computed with an impurity concentration of $n_{\mathrm{imp}}=4 \times 10^{12} \mathrm{~cm}^{-2}$ at the electron density of $n=10^{13} \mathrm{~cm}^{-2}$ while the CI/phonon-limited results are computed using the same $n_{\text {imp }}$ and phonon parameters from Ref. [5]. Our CI-limited mobility results show that a significant temperature dependence can arise even in the absence of phonon scattering.

ner oxide places the image charges in the metal closer to the SLM and screens the charged impurities more effectively. At $n=10^{12} \mathrm{~cm}^{-2}$, a 37 percent enhancement in $\mu_{\mathrm{imp}}^{\mathrm{TG}}$ can be achieved by reducing $t_{\mathrm{ox}}$ from 20 to $2 \mathrm{~nm}$. This implies that reducing $t_{\mathrm{ox}}$ can significantly mitigate the effects of charged impurities especially when $T \gg T_{F}$.

\section{FURTHER DISCUSSION AND SUMMARY}

The underlying physics of our findings stems from the transition of the 2DEG in SLM from degeneracy to nondegeneracy at higher accessible temperatures. At high temperatures $\left(T \gtrsim T_{F}\right)$, charge screening within the 2DEG becomes weaker with increasing temperature, and the charged impurity-limited mobility becomes more dependent on screening by the dielectric environment of the SLM. The non-degeneracy-to-degeneracy transition also explains why the mobility enhancement is not seen in top-gated SLG, the question posed at the beginning the paper. The linear band structure of SLG ensures that it remains degenerate even at room temperature. For example, the Fermi temperature in SLG exceeds 1300 $\mathrm{K}$ at $n=10^{12} \mathrm{~cm}^{-2}$ whereas the corresponding Fermi temperature in SLM is $29 \mathrm{~K}$. Thus, charge screening within SLG is effectively temperature-independent and dominates the screening of charged impurities at accessible temperatures. On the other hand, charge screening within SLM weakens with temperature and allows screening by the dielectric environment to play a bigger role at high temperatures.

We also point out that mobility enhancement has been observed in top-gated epitaxial SLG [33, 34]. However, it is known that the band structure of epitaxial graphene is unlike that of ideal exfoliated SLG as a result of the formation of a substrate-induced band gap [35, 36]. Assuming that electron transport in epitaxial SLG is limited by CI scattering, the low mobility in epitaxial SLG (relative to exfoliated SLG) suggests that its intrinsic charge screening is weakened, possibly from the aforementioned band structure modification.
With regard to our results, we have calculated the charged impurity-limited mobility $\left(\mu_{\mathrm{imp}}\right)$ in SLM with electron density and temperature-dependent screening. Our results agree with the several-fold improvement in room-temperature mobility reported in Refs. [8] and 15] when a high- $\kappa$ overlayer is introduced, and they are consistent with the weak charge screening found in Ref. 25]. We have found that $\mu_{\text {imp }}$ decreases with increasing temperature primarily as a result of temperature-dependent polarizability, suggesting that this temperature-dependent phenomenon is not necessarily a signature of phonon scattering. Our model also qualitatively reproduces the change in the temperature scaling of $\mu_{e}$ when $\mathrm{HfO}_{2}$ is deposited on SLM [8]. However, we are unable to reproduce accurately the magnitude and temperature-scaling exponent $\gamma$ of the mobility in our model, even with the inclusion of intrinsic phonons. This suggests that other scattering mechanisms, possibly remote phonons [10, 12, 13, 37], must be accounted for in a more realistic model of electron transport in bare and top-gated SLM. Lastly, we have shown that a thinner top oxide can lead to a significant improvement in $\mu_{\mathrm{imp}}$ at low electron densities for temperatures greater than the Fermi temperature. Our results highlight a possible strategy to optimize the device geometry for superior electron transport properties in single-layer $\mathrm{MoS}_{2}$ and other transition metal dichalcogenides.

We gratefully acknowledge the support provided by Texas Instruments, the Semiconductor Research Corporation (SRC), the South-West Academy of Nanotechnology (SWAN) under Task 4.3 Theme 2400.011, and Samsung Electronics Ltd.

* Electronic address: max.fischetti@utdallas.edu

[1] K. Novoselov, A. Geim, S. Morozov, D. Jiang, Y. Zhang, S. Dubonos, I. Grigorieva, and A. Firsov, Science 306, 666 (2004). 

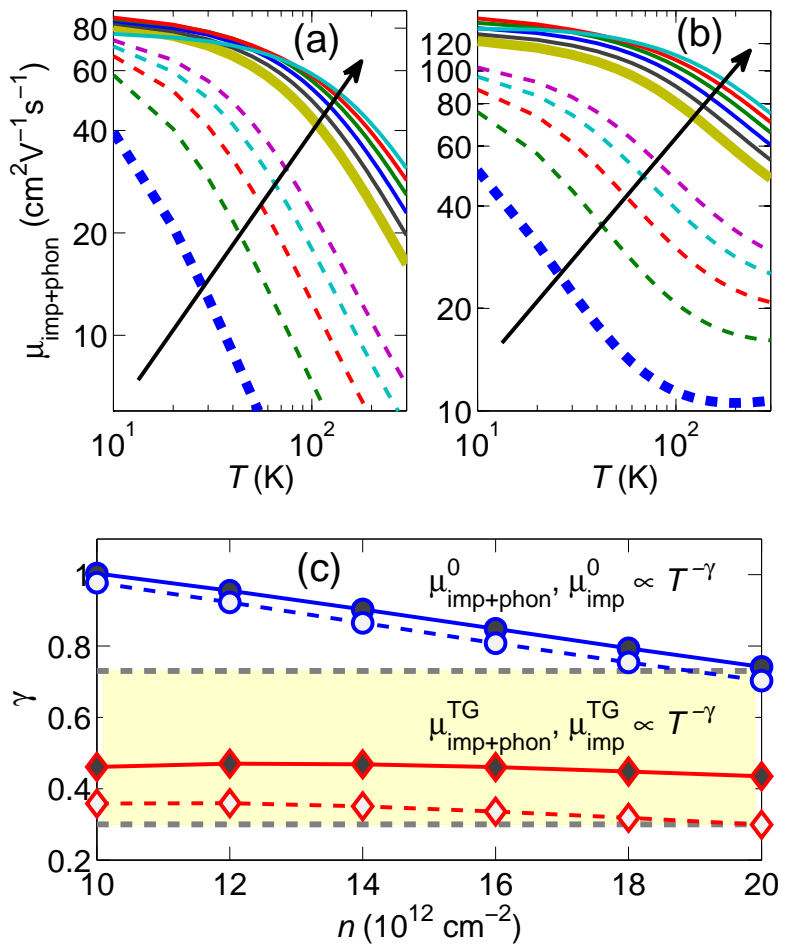

Figure 5: (Color online) Plot of (a) $\mu_{\mathrm{imp}+\mathrm{phon}}^{0}$ and (b) $\mu_{\mathrm{imp}+\text { phon }}^{\mathrm{TG}}$ for $n=10^{12}$ to $5 \times 10^{12} \mathrm{~cm}^{-2}$ in steps of $\Delta n=10^{12}$ $\mathrm{cm}^{-2}$ (dashed lines) and $n=10^{13}$ to $2 \times 10^{13} \mathrm{~cm}^{-2}$ in steps of $\Delta n=2 \times 10^{12} \mathrm{~cm}^{-2}$ (solid lines) from $T=10$ to $300 \mathrm{~K}$. The arrows indicate the direction of increasing $n$. The thick dashed (solid) line corresponds to $n=10^{12} \mathrm{~cm}^{-2}\left(10^{13} \mathrm{~cm}^{-2}\right)$. As $n$ increases, $\left|d \mu_{\mathrm{imp}+\text { phon }} / d T\right|$ becomes smaller. (c) Plot of the exponent $\gamma$ for $\mu_{\mathrm{imp}+\text { phon }}^{0}$ (solid circles), $\mu_{\mathrm{imp}}^{0}$ (open circles), $\mu_{\mathrm{imp}+\text { phon }}^{\mathrm{TG}}$ (solid diamonds) and $\mu_{\mathrm{imp}}^{\mathrm{TG}}$ (open diamonds) from fitting to $\mu_{e} \propto T^{-\gamma}$ over the range $T=200$ to $300 \mathrm{~K}$. The shaded region bounded by the dashed lines covers the range of $\gamma$ values ( 0.3 to 0.73 ) extracted for top-gated SLM in Ref. [8].

[2] Q. Wang, K. Kalantar-Zadeh, A. Kis, J. Coleman, and M. Strano, Nature Nanotechnology 7, 699 (2012).

[3] B. Radisavljevic, A. Radenovic, J. Brivio, V. Giacometti, and A. Kis, Nature Nanotechnology 6, 147 (2011).

[4] Y. Yoon, K. Ganapathi, and S. Salahuddin, Nano Lett. 11, 3768 (2011).

[5] K. Kaasbjerg, K. S. Thygesen, and K. W. Jacobsen, Phys. Rev. B 85, 115317 (2012).

[6] M.-W. Lin, L. Liu, Q. Lan, X. Tan, K. S. Dhindsa, P. Zeng, V. M. Naik, M. M.-C. Cheng, and Z. Zhou, J. Phys. D: Appl. Phys. 45, 345102 (2012).

[7] J. Lin, J. Zhong, S. Zhong, H. Li, H. Zhang, and W. Chen, Appl. Phys. Lett. 103, 063109 (2013).

[8] B. Radisavljevic and A. Kis, Nat. Mater. 12, 815 (2013).

[9] S. Adam, E. Hwang, E. Rossi, and S. Das Sarma, Solid State Commun. 149, 1072 (2009).

[10] M. V. Fischetti, D. A. Neumayer, and E. A. Cartier, J. Appl. Phys. 90, 4587 (2001).

[11] S. Fratini and F. Guinea, Phys. Rev. B 77, 195415 (2008).

[12] Z.-Y. Ong and M. V. Fischetti, Phys. Rev. B 86, 165422

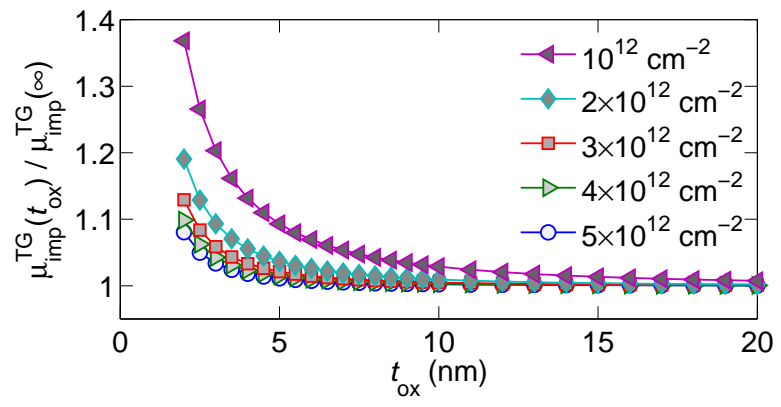

Figure 6: (Color online) Plot of $\mu_{\mathrm{imp}}^{\mathrm{TG}}\left(t_{\mathrm{ox}}\right) / \mu_{\mathrm{imp}}^{\mathrm{TG}}(\infty)$ for $n=$ $10^{12}$ to $5 \times 10^{12} \mathrm{~cm}^{-2}$ and $t_{\mathrm{ox}}=2$ to $20 \mathrm{~nm}$ at $300 \mathrm{~K}$.

(2012).

[13] Z.-Y. Ong and M. V. Fischetti, Phys. Rev. B 86, 199904(E) (2012).

[14] Z.-Y. Ong and M. V. Fischetti, Appl. Phys. Lett. 102, 183506 (2013).

[15] M. Amani, M. L. Chin, A. G. Birdwell, T. P. O'Regan, S. Najmaei, Z. Liu, P. M. Ajayan, J. Lou, and M. Dubey, Appl. Phys. Lett. 102, 193107 (2013).

[16] B. Fallahazad, S. Kim, L. Colombo, and E. Tutuc, Appl. Phys. Lett. 97, 123105 (2010).

[17] Z.-Y. Ong and M. V. Fischetti, Phys. Rev. B 86, 121409 (2012)

[18] M. Lemme, T. Echtermeyer, M. Baus, B. Szafranek, J. Bolten, M. Schmidt, T. Wahlbrink, and H. Kurz, Solid-State Electron. 52, 514 (2008).

[19] S. Kim, J. Nah, I. Jo, D. Shahrjerdi, L. Colombo, Z. Yao, E. Tutuc, and S. Banerjee, Appl. Phys. Lett 94, 062107 (2009).

[20] J. Pezoldt, C. Hummel, A. Hanisch, I. Hotovy, M. Kadlecikova, and F. Schwierz, Phys. Status Solidi C 7, 390 (2010).

[21] K. Zou, X. Hong, D. Keefer, and J. Zhu, Phys. Rev. Lett. 105, 126601 (2010).

[22] J. L. Xia, F. Chen, P. Wiktor, D. K. Ferry, and N. J. Tao, Nano Lett. 10, 5060 (2010).

[23] M. S. Fuhrer and J. Hone, Nature Nanotechnology 8, 146 (2013).

[24] B. Baugher, H. O. H. Churchill, Y. Yang, and P. JarilloHerrero, Nano Lett. 13, 4212 (2013).

[25] S. Ghatak, A. N. Pal, and A. Ghosh, ACS Nano 5, 7707 (2011).

[26] J. H. Davies, The Physics of Low-Dimensional Semiconductors (Cambridge University Press, Cambridge, UK, 1997).

[27] P. F. Maldague, Surf. Sci. 73, 296 (1978).

[28] F. Stern, Phys. Rev. Lett. 44, 1469 (1980).

[29] T. Ando, A. B. Fowler, and F. Stern, Rev. Mod. Phys. 54, 437 (1982).

[30] X. Li, J. T. Mullen, Z. Jin, K. M. Borysenko, M. Buongiorno Nardelli, and K. W. Kim, Phys. Rev. B 87, 115418 (2013).

[31] S. Das Sarma, Phys. Rev. B 33, 5401 (1986).

[32] D. Jariwala, V. K. Sangwan, D. J. Late, J. E. Johns, V. P. Dravid, T. J. Marks, L. J. Lauhon, and M. C. Hersam, Appl. Phys. Lett. 102, 173107 (2013).

[33] J. A. Robinson, M. LaBella, K. A. Trumbull, X. Weng, R. Cavelero, T. Daniels, Z. Hughes, M. Hollander, 
M. Fanton, and D. Snyder, ACS Nano 4, 2667 (2010).

[34] M. J. Hollander, M. LaBella, Z. R. Hughes, M. Zhu, K. A. Trumbull, R. Cavalero, D. W. Snyder, X. Wang, E. Hwang, S. Datta, and J. A. Robinson, Nano Lett. 11, 3601 (2011).

[35] S. Zhou, G.-H. Gweon, A. Fedorov, P. First, W. De Heer, D.-H. Lee, F. Guinea, A. C. Neto, and A. Lanzara, Nat.
Mater. 6, 770 (2007).

[36] S. Zhou, D. Siegel, A. Fedorov, F. El Gabaly, A. Schmid, A. C. Neto, D.-H. Lee, and A. Lanzara, Nat. Mater. 7, 259 (2008).

[37] Z.-Y. Ong and M. V. Fischetti, Phys. Rev. B 88, 045405 (2013). 